\title{
Fluctuations of Transmission Distribution in Disordered Conductors
}

\author{
Yuli V. Nazarov \\ Faculteit der Technische Natuurkunde, Technische Universiteit Delft, \\ 2628 CJ Delft, The Netherlands
}

(Received 18 August 1995)

\begin{abstract}
We have developed a microscopic approach to calculate the sample-to-sample fluctuations of transmission distribution in disordered conductors. This bridges the gap between Green's function and random matrix theories (RMT) of quantum transport. The results obtained show that the correlations of transmission eigenvalues (transmissions) obey universal Dyson statistics at small separations between eigenvalues and are nonuniversal otherwise. We compute the interaction between transmissions that must be postulated in a RMT to describe correctly the diffusive quantum transport.
\end{abstract}

PACS numbers: $72.10 . \mathrm{Bg}$

The concept of universal conductance fluctuations (UCF) [1] proves to be one of the most interesting concepts in mesoscopic physics. Because of quantum interference, the conductance of a disordered coherent conductor fluctuates from sample to sample by a value of the order of $G_{Q}=e^{2} / 2 \pi \hbar$. The exhaustive qualitative description of the phenomenon has been elaborated on the basis of Green's function technique [2].

The alternative explanation of UCF has been proposed by Imry [3], who claimed universal cause of the phenomenon. The conductance of a mesoscopic sample can be expressed as a sum of the eigenvalues of the transmission matrix square, $\mathbf{T} \equiv \mathbf{t}^{\dagger} \mathbf{t}$,

$$
G=\frac{e^{2}}{\pi \hbar} \operatorname{Tr}\left(\mathbf{t}^{\dagger} \mathbf{t}\right)=\frac{e^{2}}{\pi \hbar} \sum_{n} T_{n}
$$

Based on works by Pichard [4], Imry showed that the assumption of Wigner-Dyson statistics of these transmission eigenvalues yields the correct order of magnitude of UCF.

The pioneering paper by Imry has triggered persistent attempts to formulate the theory of quantum transport as a kind of random matrix theory (RMT). This theory would produce the quantitative description of the transmission matrix being based on a few general postulates [5]. However, such a theory was doomed to fail. Green's function methods clearly showed that UCF are not precisely universal. Their magnitude depends on the shape of the sample and its dimensionality. Universal RMT would never have such input parameters.

The mystery is that the RMT can be made a working theory. It may correctly describe quasi-one-dimensional geometry [6,7] and chaotic cavities [8]. Why? How could it be that the same phenomenon combines universal and nonuniversal features? Are there ways to an improved RMT which would enable us to describe a concrete physical situation?

Surprisingly enough, these questions were tackled $[9,10]$ exclusively within the RMT itself without the aid of reliable microscopic methods. This made a wide gap be- tween microscopically based theories of quantum transport and RMT.

We answer the above questions checking the Imry conjecture by the recently formulated [11] Green's function method. The short answer may be formulated as follows: correlations between transmission eigenvalues obey Wigner-Dyson statistics provided their separation is much smaller than unity, while at the separations of the order of unity the correlations become dependent on a concrete geometry of a sample. The fluctuation of conductance is contributed by both small and moderate separations. This is why the final answer combines universal and sampledependent features. We compute the effective interaction between transmissions that corresponds to the sampledependent correlations. It would be an input parameter of the imaginable improved RMT describing diffusive transport in realistic conditions.

One can draw a parallel between statistics of transmission eigenvalues through an open system and statistics of energy levels in a closed system. The latter was shown $[12,13]$ to be universal at small energy separation and sample dependent at energy differences $\equiv E_{\mathrm{th}}$.

To proceed, we make use of the multicomponent Green's function method proposed in [11]. It expresses a generating function of momenta of the transmission distribution

$$
F(\Phi)=\operatorname{Tr}\left(\frac{\mathbf{T}}{1-\sin ^{2}(\Phi / 2) \mathbf{T}}\right)
$$

in terms of an artificially constructed Green's function which is a $2 \times 2$ matrix. The Green's function introduced can be readily averaged over impurity configurations using the conventional technique [14].

To find the correlations between transmissions, we have to compute the average product of two Green's functions of the kind. The technical details will be reported elsewhere [15]. The corresponding diagrams preserve the common Cooperon diffusion structure [1]. However, those are difficult to handle due to the extra matrix structure and coordinate dependence of the average Green's function. The 
compact answer is only possible to obtain for the expressions of the form (2). Using a common diagrammatic trick outlined in [16], we obtain

$$
\sin \Phi \sin \Phi^{\prime}\left\langle F(\Phi) F\left(\Phi^{\prime}\right)\right\rangle=4 \frac{\partial}{\partial \Phi} \frac{\partial}{\partial \Phi^{\prime}}\left[\ln \operatorname{det}\left(\breve{1}-\check{K}^{d}\right)+\ln \operatorname{det}\left(\check{1}-\check{K}^{c}\right)\right] .
$$

The operator $\check{K}^{d(c)}$ is actually an integral kernel corresponding to a diffusion (Cooperon) ladder section. For a common case of contact impurity potential characterized by the elastic scattering time $\tau$ it reads

$$
\begin{aligned}
K_{a b, c d}^{d}\left(x, x^{\prime} ; \Phi, \Phi^{\prime}\right)= & \frac{1}{\tau(x)}\left\langle G^{a c}\left(x, x^{\prime} ; \Phi\right)\right\rangle \\
& \times\left\langle G^{a d}\left(x, x^{\prime} ; \Phi^{\prime}\right)\right\rangle, \\
K_{a b, c d}^{c}\left(x, x^{\prime} ; \Phi, \Phi^{\prime}\right)= & \frac{1}{\tau(x)}\left\langle G^{a c}\left(x, x^{\prime} ; \Phi\right)\right\rangle \\
& \times\left\langle G^{d a}\left(x^{\prime}, x ; \Phi^{\prime}\right)\right\rangle,
\end{aligned}
$$

with indices labeling spin and the additional matrix structure.

To solve the problem, we have to find the eigenvalues of the operators $\breve{1}-\check{K}^{d(c)}, \mu$. It is possible to derive diffusionlike equations for those. These equations can be solved for a slab geometry of the conductor. In this case $\mu$ can be readily expressed in terms of common diffusion and Cooperon mode eigenvalues.

These modes have been intensively studied [14], and I remind the reader the results in short. Let the slab dimensions be $L_{x}, L_{y}, L_{z}, x$ being transport direction. First, the discrete diffusion and Cooperon modes can be labeled by three integer numbers $n_{x, y, z}, n_{y, z}$ ranging from 0 to $\infty$ and $n_{x}$ starting from 1 . The eigenvalues normalized by Thouless energy $D / L_{x}^{2}$ are given by $\left[\vec{n}=\left(n_{x}, n_{y}, n_{z}\right)\right]$

$$
\boldsymbol{\epsilon}_{\vec{n}}=\pi^{2}\left[n_{x}^{2}+\left(\frac{n_{y} L_{x}}{L_{y}}\right)^{2}+\left(\frac{n_{z} L_{x}}{L_{y}}\right)^{2}\right] .
$$

It is easy to take into account spin effects and dephasing by magnetic field. To describe those, we introduce the following dimensionless parameters: $\eta_{\mathrm{SO}}=D \tau_{\mathrm{SO}} / L_{x}^{2}, \tau_{\mathrm{SO}}$ being characteristic time of the spin-flip process, and $\eta=$ $e H L_{x} L_{y} / c \hbar, H$ being magnetic field $\| z$. We can take different $\eta$ for two different Green's functions to average, thus describing parametric correlations of the transmission fluctuations. Then all diffusion modes acquire an extra shift $\left(\eta-\eta^{\prime}\right)^{2}$ while the Cooperon ones are shifted by $\left(\eta+\eta^{\prime}\right)^{2}$. If the spin-orbit interaction is taken into account, for each branch there are three degenerate eigenvalues corresponding to spin triplet and one corresponding to spin singlet. The triplet eigenvalues acquire the same extra shift for all branches, $\epsilon^{\text {triplet }}=\epsilon^{\text {singlet }}+\eta_{\text {SO }}$.

Because of the extra matrix structure of the Green's functions in use there are two eigenvalues $\mu$ corresponding to each Cooperon or diffusion mode,

$$
\mu_{m}^{ \pm}=\frac{l^{2}}{L_{x}^{2}}\left(\epsilon_{m}-\frac{\left(\Phi \pm \Phi^{\prime}\right)^{2}}{4}\right)
$$

where mean free path $l=\sqrt{D \tau}, m$ labeling all above mentioned modes. Substituting this into (3), we find

$$
\sin \Phi \sin \Phi^{\prime}\left\langle F(\Phi) F\left(\Phi^{\prime}\right)\right\rangle=4 \frac{\partial}{\partial \Phi} \frac{\partial}{\partial \Phi^{\prime}} \sum_{\text {modes }}\left[\ln \left(\epsilon_{m}-\frac{\left(\Phi+\Phi^{\prime}\right)^{2}}{4}\right)+\ln \left(\epsilon_{m}-\frac{\left(\Phi+\Phi^{\prime}\right)^{2}}{4}\right)\right] .
$$

Finally we express the answer in terms of a density-density correlator. It is convenient to consider the density in new coordinate $\lambda$ related to transmissions by $T=1 / \sinh ^{2} \lambda$. Using the identity $2 \pi \rho(\lambda)=F(2 \lambda+\pi)-F(2 \lambda-\pi)$ that immediately follows from (2), we obtain

$$
\begin{aligned}
\left\langle\rho(\lambda, \eta) \rho\left(\lambda^{\prime}, \eta^{\prime}\right)\right\rangle & =\frac{1}{2 \pi^{2}} \sum_{\text {modes }} Z\left(\sqrt{\epsilon_{m}}+\pi\right)+Z\left(\sqrt{\epsilon_{m}}-\pi\right)-2 Z\left(\sqrt{\epsilon_{m}}\right), \\
Z\left(x ; \lambda, \lambda^{\prime}\right) & \equiv \operatorname{Re}\left(\frac{1}{\left[x+i\left(\lambda+\lambda^{\prime}\right)\right]^{2}}+\frac{1}{\left[x+i\left(\lambda-\lambda^{\prime}\right)\right]^{2}}\right) .
\end{aligned}
$$

This relation provides an exact microscopic answer for transmission correlations and is the main result of the present work. It describes the effects of dimensionality and crossovers between different statistical ensembles.

Let us now analyze the physically meaningful consequences of (9).

First let us consider correlations at small separations between $\lambda^{\prime}$ s, $\left|\lambda^{\prime}-\lambda\right| \ll 1$. Then the sum is dominated by the second term corresponding to $n_{x}=1, n_{y}=n_{z}=$ 0 . Manipulating $\eta, \eta_{\text {SO }}$ allows us to consider limits of different pure statistical ensembles and to reconcile that

$$
\left\langle\rho(\lambda) \rho\left(\lambda^{\prime}\right)\right\rangle=-\frac{s^{2}}{\pi^{2} \beta} \operatorname{Re} \frac{1}{\left(\lambda-\lambda^{\prime}+i 0\right)^{2}},
$$

$s$ being an extra degeneracy of transmission eigenvalues not lifted by the random scattering. This coincides with a known result of Dyson [17] provided $\left|\lambda-\lambda^{\prime}\right|$ exceeds the average spacing between transmissions, $s G_{Q} / G$, and thus proves Imry's conjecture [3].

The present theory is not able to give a detailed form of the correlator at separations comparable with the average spacing, albeit we believe it coincides with Dyson 
expressions. This is a common drawback of theories perturbative in $G / G_{q}$, for instance, [12]. At small separations, the fluctuations of the mode $n_{x}=1, n_{y, z}=0$ become big, like the fluctuations of the mode $n_{x, y, z}$ for an isolated system [12]. For an isolated system, the answer has been gotten by the nonperturbative supersymmetry technique [13]. The development of the supersymmetry technique for the diagrammatic approach [11] that has been started [18] recently will do the same for transmission.

If $\eta \neq \eta^{\prime} \ll \pi$, the contribution of divergent mode,

$$
\begin{aligned}
\left\langle\rho(\lambda, \eta) \rho\left(\lambda^{\prime}, \eta^{\prime}\right)\right\rangle= & -\frac{s^{2}}{2 \pi^{2}} \\
& \times \operatorname{Re} \frac{1}{\left[\lambda-\lambda^{\prime}+i\left(\eta-\eta^{\prime}\right) / 2 \pi\right]^{2}},
\end{aligned}
$$

reconciles universal parametric correlations [19] that correspond to Brownian motion of transmissions [20].

Thus we have shown that the transmission statistics at small separations appears to be the universal WignerDyson one arising from a term corresponding to the lowest diffusion (Cooperon) mode. The rest of the terms in (9), however, do not have universal form. This explains why the fluctuations of the quantities integrated over transmission distribution, like conductance, do not obey universal relations.

The question of interest is if an improved RMT can ever account for details of the sample geometry and describe crossover between ensembles. The answer seems to be positive.

To see this let us first check the so-called DorokhovMello-Pereyra-Kumar (DMKP) method [9] that seems to describe correctly quasi-one-dimensional systems [7]. For the quasi-one-dimensional case, and for pure ensembles, $\sqrt{\epsilon_{n}}=\pi n$, the sum in (9) becomes telescopic and can be readily done. The result indeed coincides with the one obtained in [6] by the Dorokhov-Mello-Pereyra-Kumar method,

$$
\begin{aligned}
\left\langle\rho(\lambda) \rho\left(\lambda^{\prime}\right)\right\rangle=\frac{s^{2}}{\pi^{2} \beta} \operatorname{Re}[ & \frac{1}{\left(\lambda-\lambda^{\prime}+i \pi\right)^{2}} \\
& \left.-\frac{1}{\left(\lambda-\lambda^{\prime}+i 0\right)^{2}}\right],
\end{aligned}
$$

To proceed further, let us note that the correlator (9) possesses the following property,

$$
\left\langle\rho(\lambda) \rho\left(\lambda^{\prime}\right)\right\rangle=K\left(\lambda-\lambda^{\prime}\right)+K\left(\lambda+\lambda^{\prime}\right) .
$$

In the Green's function framework, it is impossible to find a physical reason for this. RMT gives a bright pattern to comprehend such a symmetry [6]: consider a system of classical particles confined in the region $0<\lambda$. The correlator of such a form emerges if one assumes pair interaction $U\left(\lambda-\lambda^{\prime}\right)$ between the particles and their "image charges" mirroring the particles at negative $\lambda$. There is a simple relation between Fourier components of the interaction and $K[6]$ :

$$
U(k)=\frac{s^{2}}{\beta K(k)} .
$$

This enables one to express the transmission correlations in terms of an effective interaction between eigentransmissions. This interaction shall be computed from (9) for a given sample geometry. A RMT that postulates this interaction will give a quantitatively correct picture of quantum transport for this geometry.

In Fig. 1 I have plotted the Fourier component of the interaction for a pure ensemble and for different geometries (wire, $L_{x} \gg L_{z, y}$; square, $L_{x}=L_{y} \gg L_{z}$; and cube, $L_{x}=L_{y}=L_{z}$ ) along with Wigner-Dyson interaction. At moderately large $k$ (small separations) for all geometries interaction rapidly converges to Wigner-Dyson values, whereas its small $k$ behavior drastically differs. For a wire, $U(k) \rightarrow k^{-2}$, and the eigenvalue repulsion exceeds the Wigner-Dyson one. For 2D geometry, $U(k) \rightarrow$ $2 L_{x} / L_{y}|k|$. For the $3 \mathrm{D}$ case, $U(k) \rightarrow \pi L_{x}^{2} / L_{y} L_{z}+c|k|$. This corresponds to the following asymptotes at big $\lambda$ :

$$
\begin{aligned}
U(\lambda) \rightarrow-|\lambda| & \text { for } 1 \mathrm{D}, \\
U(\lambda) \rightarrow-2 L_{x} / \pi L_{y} \ln |\lambda| & \text { for } 2 \mathrm{D}, \\
U(\lambda) \rightarrow 2\left(L_{x} / L_{y}+L_{x} / L_{z}-1\right) / \lambda^{2} & \text { for } 3 \mathrm{D} .
\end{aligned}
$$

It is interesting to note that in $3 \mathrm{D}$ the asymptotical interaction can be attractive for sufficiently wide slabs.

In conclusion, we have microscopically checked Imry's conjecture [3] for transmission matrix explicitly showing where and why the Wigner-Dyson approach does not work. We have calculated the interaction that shall be

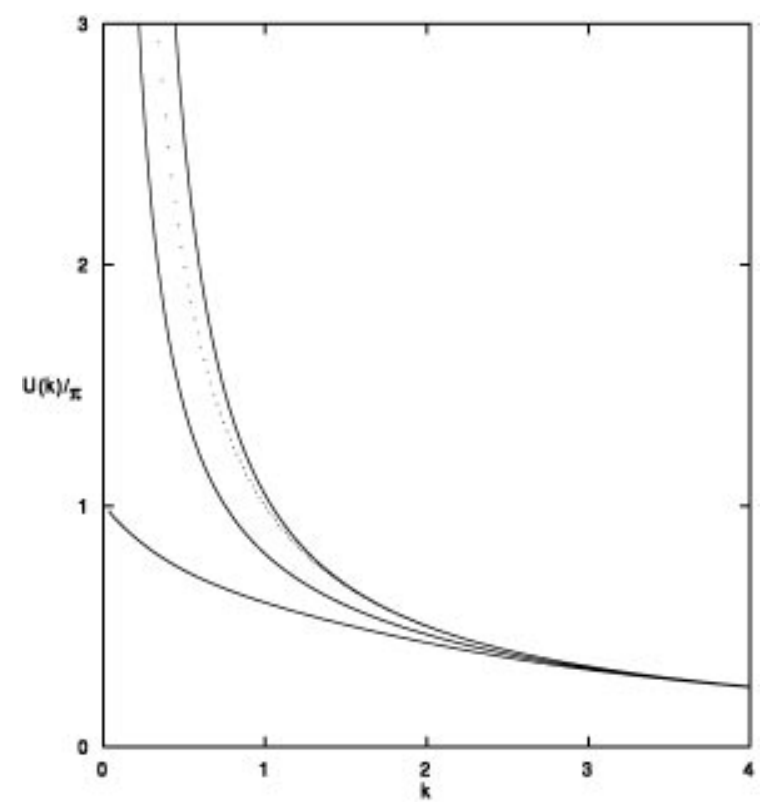

FIG. 1. The Fourier component of effective eigenvalue interaction for (from the uppermost to lowest curve) wire, the Wigner-Dyson interaction, square, and cube. 
postulated in an imaginable RMT that describes realistic diffusive conductors.

I am indebted to C. W. J. Beenakker for his persistent interest in this work, teaching me RMT, and making many instructive suggestions. I appreciate interesting discussions of the results with G.E. W. Bauer, K. Frahm, and $\mathrm{B}$. Rejaei. This work is a part of the research program of the "Stichting voor Fundamenteel Onderzoek der Materie" (FOM), and I acknowledge the financial support from the "Nederlandse Organisatie voor Wetenschappelijk Onderzoek" (NWO).

[1] B. L. Altshuler, Pis'ma Zh. Eksp. Teor. Fiz. 41, 530 (1985) [JETP Lett. 41, 648 (1985)]; P. A. Lee and A. D. Stone, Phys. Rev. Lett. 35, 1622 (1985); B. L. Altshuler and D. E. Khmelnitskii, JETP Lett. 42, 559 (1985).

[2] P. A. Lee, A. D. Stone, and H. Fukuyama, Phys. Rev. B 35, 1039 (1987).

[3] Y. Imry, Europhys. Lett. 1, 249 (1986).

[4] J.-L. Pichard, thesis, University of Paris, Orsay [Inst. Report No. 2858, 1984].

[5] A. D. Stone, P. A. Mello, K. A. Muttalib, and J.-L. Pichard, in Mesoscopic Phenomena in Solids, edited by L. Alt- shuler, P. A. Lee, and R. A. Webb (North-Holland, Amsterdam, 1991).

[6] C. W. J. Beenakker and B. Rejaei, Phys. Rev. B 49, 7499 (1994).

[7] K. Frahm, Phys. Rev. Lett. 74, 4706 (1995).

[8] H. U. Barranger and P. A. Mello, Phys. Rev. Lett. 73, 142 (1994); R. A. Jalambert, J.-L. Pichard, and C. W. J. Beenakker, Europhys. Lett. 27, 255 (1994).

[9] O. N. Dorokhov, Solid State Commun. 51, 381 (1984); P. A. Mello, P. Pereyra, and N. Kumar, Ann. Phys. (N.Y.) 181, 290 (1988).

[10] S. Iida, H. A. Weidenmueller, and J. A. Zuk, Ann. Phys. (N.Y.) 200, 219 (1990).

[11] Yu. V. Nazarov, Phys. Rev. Lett. 73, 134 (1994).

[12] B. L. Altshuler and B. I. Shklovskii, Zh. Eksp. Teor. Fiz. 91, 220 (1986) [Sov. Phys. JETP 64, 127 (1986)].

[13] K. B. Efetov, Adv. Phys. 32, 53 (1983).

[14] P. A. Lee and T. V. Ramakrishnan, Rev. Mod. Phys. 57, 287 (1987).

[15] Yu. V. Nazarov (to be published).

[16] Yu. V. Nazarov, Phys. Rev. B 52, 4720 (1995).

[17] F. J. Dyson, J. Math. Phys. (N.Y.) 3, 1191 (1962).

[18] B. Rejaei (to be published).

[19] B. D. Simons, P. A. Lee, and B. L. Altshuler, Phys. Rev. Lett. 70, 4122 (1993).

[20] C. W. J. Beenakker, Phys. Rev. Lett. 70, 4126 (1993). 